\title{
Primeiro registro de Amblyomma geayi (Acari: ixodidae) em preguiça (Bradypus variegatus) no estado do Acre, Amazônia Ocidental: relato de caso
}

\author{
[First record of amblyomma geayi (acari: ixodidae) in sloth (bradypus variegatus) in the Acre state, \\ Western Amazon: case report]

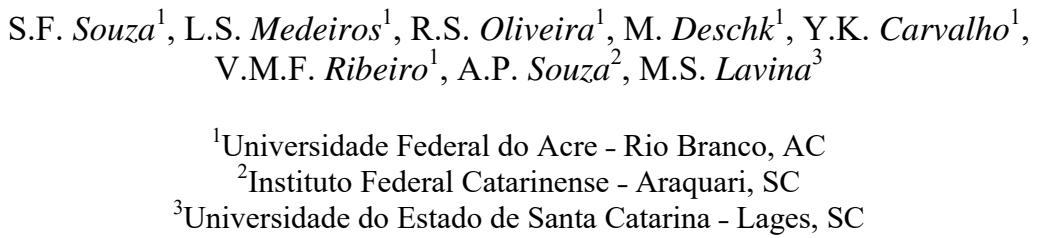

\section{RESUMO}

O Amblyomma geayi é um carrapato da família Ixodidae, encontrado principalmente em mamíferos e aves silvestres de regiões tropicais da América do Sul. Descreve-se a ocorrência de um carrapato ixodídeo da espécie A. geayi, encontrado em uma preguiça (Bradypus variegatus) proveniente do Parque Zoobotânico da Universidade Federal do Acre, Rio Branco, Acre, Amazônia Ocidental.

Palavras-chave: carrapatos, Ixodídeos

\begin{abstract}
Amblyomma geayi is a tick of the Ixodidae family found primarily in mammals and wild birds from tropical regions in South America. This case report the occurrence of an ixodid tick species A. geayi found in a brown-throated sloth (Bradypus variegatus) from the botanical zoo Park of the Federal University of Acre, Rio Branco, Acre, Western Amazon.
\end{abstract}

Keywords: ticks, Ixodid

\section{INTRODUÇÃO}

O estado do Acre está inserido em uma das regiões mais ricas, em termos de biodiversidade, na Amazônia Ocidental (Calouro, 1999). Para que essa biodiversidade seja mantida, é necessário o conhecimento de possíveis fatores que possam causar enfermidades nesses animais, incluindo a presença de ectoparasitas que, além da espoliação sanguínea, podem servir de vetores de patógenos de diversas doenças.

Apesar de algumas características importantes, como a história demográfica, a densidade populacional, os padrões de migração e a estrutura da população em algumas espécies de preguiças, ainda serem desconhecidas (MoraesBarros et al., 2006), sabe-se que as preguiças (Bradypus tridactylus e B. variegatus) são vistas em florestas primárias, secundárias, matas de várzeas e até mesmo de inundação (igapós), como também em ambientes degradados com proliferação de imbaubeiras (Miranda e Costa, 2007).

A preguiça comum, ou Bradypus variegatus, pode ser encontrada na região Amazônica, incluindo a Amazônia Ocidental, por possuir hábitos arborícolas (Calouro, 1999) e ampla distribuição, ocorrendo nas Américas Central e do Sul, desde Costa Rica, Equador, Colômbia, Venezuela, Peru, Bolívia, Argentina até praticamente todo o Brasil (Miranda e Costa, 2007). No Brasil, sua presença tem sido relatada também em áreas de Mata Atlântica no Rio de Janeiro (Modesto et al., 2008), Espírito Santo (Vale e Pereira, 2015), São Paulo, Bahia e Minas Gerais (Moraes-Barros et al., 2006). Apesar de ser considerada não ameaçada, Silva (2013) 
enfatizou a necessidade de medidas de conservação populacional para a espécie.

Carrapatos do gênero Amblyomma spp. têm se tornado motivo de estudos na região Amazônica. Em 40,2\% de aves silvestres capturadas no Pará foram encontrados carrapatos de diferentes espécies de Amblyomma spp.; o mais encontrado, no entanto, foi a espécie Amblyomma geayi, com sete larvas e uma ninfa (Ogrzewalska et al., 2010). Em outro estudo semelhante, também no Pará, $3,1 \%$ das aves silvestres capturadas apresentaram carrapatos, sendo eles $A$. calcaratum, A. geayi, A. humerale e A. longirostre (Martins et al., 2014).

O A. geayi também foi encontrado por Martins et al. (2013) em B. variegatus, em Belém, estado do Pará. Em Vilhena, Rondônia, foram encontradas diferentes espécies de Amblyomma spp. em capivara, Hydrochoerus hydrochaeris (Linnaeus), águia-harpia, Harpia harpyja (Linnaeus), e jabuti, Chelonoidis denticulada (Linnaeus). Nesse estudo, Labruna et al. (2010) registraram pela primeira vez a presença de $A$. romitii, porém a presença do $A$. geayi não foi relatada. Ainda em Rondônia, município de Monte Negro, A. humerale foi encontrado parasitando Geochelone denticulada e $G$. carbonária, espécies de jabutis encontradas no leste e no sul da Floresta Amazônica (Labruna et al., 2002). Não foram encontrados registros da presença de Amblyomma spp. no estado do Acre. Diante disso, é de grande importância a descrição e a realização de estudos sobre a ocorrência das espécies deste ixodídeo na Amazônia Ocidental.

\section{CASUÍSTICA}

Em outubro de 2014, um animal jovem da espécie B. variegatus (Fig. 1A e 1B), fêmea, foi encontrado no Parque Zoobotânico da
Universidade Federal do Acre e encaminhado à Unidade de Ensino e Pesquisa em Medicina Veterinária da Universidade Federal do Acre, no município de Rio Branco, Acre (latitude: -9.974, longitude: -67.8076) por apresentar um pequeno ferimento no terceiro dígito do membro pélvico direito de aproximadamente $3 \mathrm{~mm}$ de largura por $5 \mathrm{~mm}$ de comprimento. Durante a avaliação física, constatou-se que a preguiça apresentava bom estado de saúde dentro das características da espécie, com nível de consciência alerta. A contenção física foi realizada conforme as recomendações de Miranda e Costa (2007), que citaram a fácil manipulação por meio da contenção pelas costas ou pelas extremidades dos braços. Além disso, foi realizada a inspeção manual em busca de possíveis alterações sob os pelos, onde foi encontrado um carrapato do gênero Amblyomma spp. (Fig. 1B) na região do pavilhão auricular esquerdo, que foi removido com auxílio de pinça e conservado em álcool $70 \%$ para posterior identificação. Após limpeza da ferida com solução de cloreto de sódio a 0,9\%, o Corpo de Bombeiros foi acionado para que fizesse o encaminhamento do animal até o Centro de Triagem de Animais Silvestres Cetas/Ibama, localizado no Parque Ambiental Chico Mendes, para providências de reintrodução.

O espécime (Fig. 2) foi identificado com auxílio de estereomicroscópio da marca Leica, modelo S8APO, com aumento de 8 a $35 \mathrm{x}$ e software específico Leica Application Suit, versão 3.0, para inclusão de escala milimétrica. Para a identificação do ixodídeo, utilizou-se a chave dicotômica de Barros-Battesti et al. (2006). O exemplar macho apresenta sulco marginal completo, placas ventrais e o primeiro par de coxas com dois espinhos curtos, sendo o interno muito reduzido. 


\section{Primeiro registro de...}

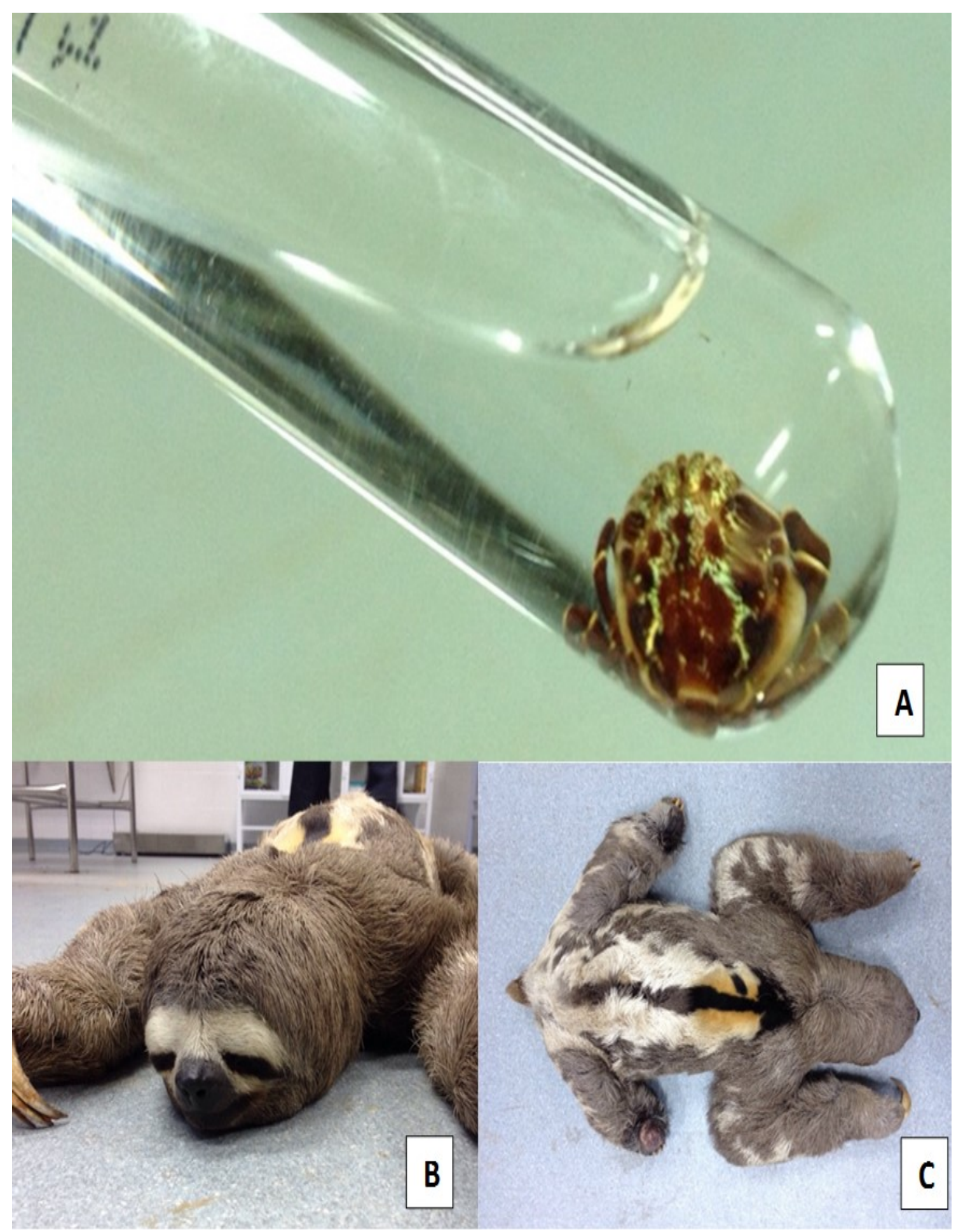

Figura 1. Amblyomma spp. (A) encontrado em Bradypus variegatus (B e C) atendida na Unidade de Ensino e Pesquisa de Medicina Veterinária da Universidade Federal do Acre, Rio Branco, Acre, Brasil. 

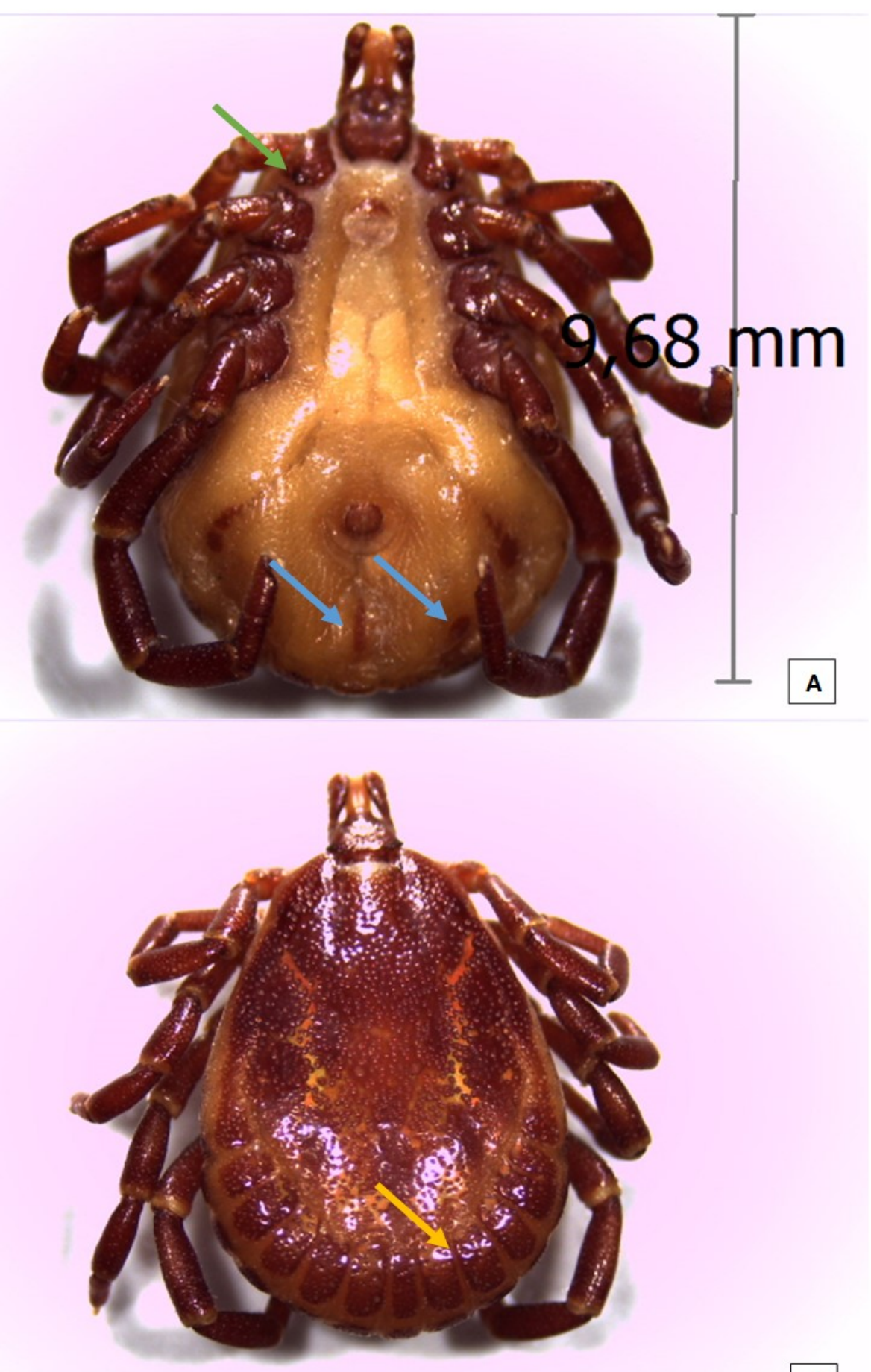

Figura 2. Macho de Ambyomma geagi fotografado em estereomicroscópico com aumento de 8x. A: vista ventral destacando as placas ventrais (setas azuis) e coxa I com dois espinhos curtos (seta verde), sendo o interno muito reduzido; B: vista dorsal destacando o sulco marginal completo (seta amarela). 


\section{DISCUSSÃO}

A presença do gênero Amblyomma spp. tem sido descrita em diferentes espécies da região Amazônica, como em aves silvestres na Amazônia Ocidental, Pará (Ogrzewalska et al., 2010; Martins et al., 2014), quelônios (Labruna et al., 2002), Hydrochoerus hydrochaeris, capivara, Harpia harpyja, águia-harpia, e jabuti, Chelonoidis denticulada, em Rondônia, na Amazônia Ocidental (Labruna et al., 2010) e em uma preguiça da espécie $B$. variegatus, também no Pará (Martins et al., 2013).

Apesar de o A. geayi ser encontrado parasitando animais silvestres na região Amazônica, conforme relatado por Ogrzewalska et al. (2010) e Martins et al. (2014), mais descrições desta espécie são necessárias, uma vez que Martins et al. (2010) descreveram morfologicamente as ninfas de 27 das 29 espécies de Amblyomma spp. encontradas no Brasil, afirmando que as ninfas de A. goeldii e de A. geayi permaneciam sem descrição. A primeira descrição desta espécie no estágio de ninfa foi realizada por Martins et al. (2013), que, assim como no presente relato, encontraram o carrapato parasitando a preguiça comum (B. variegatus). A escassez de relatos dessa natureza evidencia a necessidade da realização de mais estudos sobre a distribuição de A. geayi em diferentes regiões e espécies de hospedeiros.

\section{CONCLUSÃO}

Por meio da identificação do ectoparasito, foi possível concluir que o Amblyomma geayi está presente também no estado do Acre e foi encontrado parasitando uma preguiça da espécie Bradypus variegatus.

\section{REFERÊNCIAS}

BARROS-BATTESTI, D.M.; ARZUA, M.; BECHARA, G.H. et al. Carrapatos de importância médico-veterinária da região neotropical: um guia ilustrado para identificação de espécies. São Paulo: Vox, 2006. 223p.

CALOURO, A.M. Riqueza de mamíferos de grande e médio porte do Parque Nacional da Serra do Divisor (Acre, Brasil). Rev. Bras. Zool., v.16, p.195-213, 1999.

Arq. Bras. Med. Vet. Zootec., v.68, n.4, p.953-957, 2016
LABRUNA, M.B.; BARBIERI, F.S.; MARTINS, T.F. et al. New tick records in Rondônia, western Brasilian Amazon. Rev .Bras. Parasitol. Vet., v.19, p.192-194, 2010.

LABRUNA, M.B.; CAMARGO, L.M.A.; TERRASSINI, F.A. et al. Notes on parasitismo by Amblyomma humerale (Acari: Ixodidae) in the state of Rondônia, western Amazon, Brazil. J. Med. Entomol., v.39, p.814-817, 2002.

MARTINS, T.F.; FECCHIO, A.; LABRUNA, M.B. Ticks of the genus Amblyomma (Acari: Ixodidae) on wild birds in the Brazilian Amazon. Syst. Appl. Acarol., v.19, p.385-392, 2014.

MARTINS, T.F.; ONOFRIO, V.C.; BARROSBATTESTI, D.M. et al. Nymphs of the genus Amblyomma (Acari: Ixodidae) of Brazil: descriptions, redescriptions, and identification key. Ticks Tick Borne Dis., v.1, p.75-90, 2010.

MARTINS, T.F.; SCOFIEOD, A.; OLIVEIRA, W.B.L. et al. Morphological description of the nymphal stage of Amblyomma geayi and new nymphal records of Amblyomma parkeri. Ticks Tick Borne Dis., v.4, p.181-184, 2013.

MIRANDA, F.; COSTA, A.M. Xenarthra (tamanduá, tatu, preguiça). In: CUBAS, Z.S.; SILVA, J.C.R.; CATÃO-DIAS, J.L. Tratado de animais selvagens. São Paulo: Roca, 2007. p.402-414.

MODESTO, T.C.; PESSÔA, F.S.; ENRICI, M.C. et al. Mamíferos do Parque Estadual do Desengano, Rio de Janeiro, Brasil. Biota Neotrop., v.8, p.153-159, 2008.

MORAES-BARROS, N.; SILVA, J.A.B.; MIYAKI, C.Y. et al. Comparative phylogeography of the Atlantic forest endemic sloth (Bradypus torquatus) and the widespread three-toed sloth (Bradypus variegatus) (Bradypodidae, Xenarthra). Genetica, v.126, p.189-198, 2006.

OGRZEWALSKA, M.; UEZU, A.; LABRUNA, M.B. Ticks (Acari: Ixodidae) infesting wild birds in the eastern Amazon, northern Brazil, with notes on rickettsial infection in ticks. Parasitol. Res., v.106, p.809-816, 2010.

SILVA, S.M. Contribuições para a conservação de Bradypus variegatus (preguiça comum): processos históricos e demográficos moldando a diversidade nuclear. 2013. 180f. Tese (Doutorado em Biologia) Departamento de Genética e Biologia Evolutiva do Instituto de Biociências, Universidade de São Paulo, São Paulo, SP.

VALE, V.; PEREIRA, M.C.A. Diversidade de mamíferos do Parque Estadual Cachoeira da Fumaça, Alegre, Espírito Santo. Natureza on line., v.13, p.234239, 2015. 